\title{
Feedback from AGN: The Kinetic/Radio Luminosity Function
}

\section{Gabriele Melini $^{1}$, Fabio La Franca ${ }^{1}$, and Fabrizio Fiore ${ }^{2}$}

${ }^{1}$ Dipartimento di Fisica, Università Roma Tre, Via della Vasca Navale 84, I-00146 Roma, Italy Email: melini, lafranca@fis.uniroma3.it

${ }^{2}$ INAF-Oss. Astronomico di Roma, Via Frascati 33, I-00040, Monteporzio Catone, Italy

Keywords. galaxies: active, quasars: general, radio continuum: galaxies, X-rays: galaxies

We have measured the probability distribution function of the ratio $R_{\mathrm{X}}=\log L_{1.4} / L_{\mathrm{X}}$, where $L_{1.4} / L_{\mathrm{X}}=\nu L_{\nu}(1.4 \mathrm{GHz}) / L_{\mathrm{X}}(2-10 \mathrm{keV})$, between the $1.4 \mathrm{GHz}$ and the unabsorbed $2-10 \mathrm{keV}$ luminosities and its dependence on $L_{\mathrm{X}}$ and $z$. We have used a complete sample of $\sim 1800$ hard X-ray selected AGN, observed in the $1.4 \mathrm{GHz}$ band, cross-correlated in order to exclude FR II-type objects, and thus obtain a contemporaneous measure of the radio and X-ray emission. The distribution $P\left(R_{\mathrm{X}} \mid L_{\mathrm{X}}, z\right)$ is shown in Figure 1. Convolution of the distribution $P\left(R_{\mathrm{X}} \mid L_{\mathrm{X}}, z\right)$ with the 2-10 keV X-ray AGN luminosity function from La Franca et al. (2005) and the relations between radio power and kinetic energy from Best et al. (2006) and Willott et al. (1999) allows us to derive the AGN kinetic power and its evolution. As shown in Figure 1, our results are in good agreement with the predictions of the most recent models of galaxy formation and evolution (e.g., Croton et al. 2006), where AGN radio feedback is required to quench the star formation.
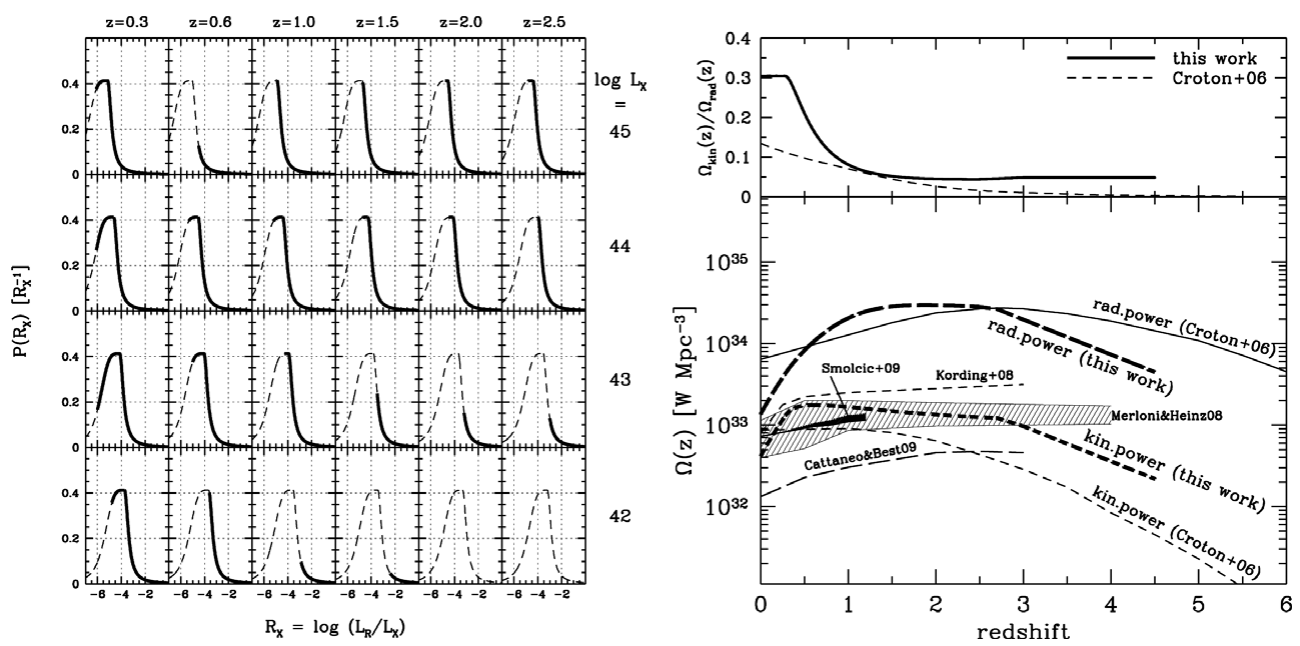

Figure 1. Left: $P\left(R_{\mathrm{X}} \mid L_{\mathrm{X}}, z\right)$ distribution in different bins of $L_{\mathrm{X}}$ and $z$. Right: Radiative and kinetic power density as a function of $z$ from this and previous works.

\section{References}

Best, P. N., Kaiser, C. R., Heckman, T. M., \& Kauffmann, G. 2006, MNRAS, 368, L67

Croton, D. J., et al. 2006, MNRAS, 365, 11

La Franca, F., et al. 2005, ApJ, 635, 864

Willott, C. J., Rawlings, S., Blundell, K. M., \& Lacy, M. 1999, MNRAS, 309, 1017 